\title{
USO DE HERBICIDAS NO CONTROLE DE PLANTAS DANINHAS E EM MUDAS PRÉ-BROTADAS (MBPS) DE DIFERENTES VARIEDADES DE CANA-DE-AÇÚCAR
}

\author{
HERBICIDES TO WEED CONTROL AND IN PRE-BUDDED SEEDLINGS (MBPS) OF DIFFERENT VARIETIES OF SUGARCANE
}

Rafael Pires Silva ${ }^{\mathrm{a}}$, Beatriz Alexandre Torres ${ }^{\mathrm{b}}$, Jose Luiz Souza $^{\mathrm{b}}$, Paulo Henrique Vieira dos Santos ${ }^{\mathrm{b}}$, Patricia Andrea Monquero ${ }^{\mathrm{c} *}$

${ }^{a}$ Bolsista da Fundação de Amparo a Pesquisa do Estado de São Paulo, Centro de Ciências Agrárias, Universidade Federal de São Carlos, São Paulo, Brasil. ${ }^{b}$ Centro de Ciências Agrárias, Universidade Federal de São Carlos, São Paulo, Brasil. 'Departamento de Recursos Naturais e Proteção Ambiental, Centro de Ciências Agrárias, Universidade Federal de São Carlos, São Paulo, Brasil.

*Autor correspondente: pamonque@hotmail.com.

\section{INFORMAÇÕES DO ARTIGO}

Histórico do artigo:

Recebido: 12 Novembro 2018.

Aceito: 30 Junho 2019.

Publicado: 08 Agosto 2019.

\section{Palavras-chave/Keywords:}

Controle Químico/Chemical Control.

Pré-Emergente/ Pre-Emergence.

Seletividade/ Selectivity.

\section{Financiamento:}

Fundação de Amparo e Pesquisa do Estado de São Paulo - FAPESP projeto 2016/05690-3.

Direito Autoral: Este é um artigo de acesso aberto distribuído sob os termos da Licença Creative Commons, que permite uso, distribuição e reprodução irrestritos em qualquer meio, desde que $\mathrm{o}$ autor e a fonte originais sejam creditados

\section{Citação deste artigo:}

SILVA, R. P.; TORRES, B. A.; SOUZA, J. L.; SANTOS, P. H. V.; MONQUERO, P. A. Uso de herbicidas no controle de plantas daninhas e em mudas pré-brotadas (MBPS) de diferentes variedades de cana-deaçúcar. Revista Brasileira de Herbicidas, v. 18, n.1. 2019.

\begin{abstract}
RESUMO
Mudas pré-brotadas (MPB) de cana-de-açúcar chegam ao campo com a parte aérea e raízes desenvolvidas podendo apresentar sensibilidade a herbicidas e mudar o manejo de controle de plantas daninhas. Portanto, os objetivos deste trabalho foram: determinar o controle químico das plantas daninhas Cynodon dactylon, Chloris polydactyla e Merremia aegyptia; e determinar o efeito dos herbicidas em mudas de pré-brotadas de diferentes variedades de cana-de-açúcar. O delineamento experimental foi inteiramente casualizado com onze tratamentos e quatro repetições. Os herbicidas saflufenacil, clomazone, metribuzim, tebuthiuron, indaziflam, saflufenacil + clomazone, saflufenacil + metribuzim, saflufenacil + tebuthiuron, indaziflam + metribuzin, indaziflam + tebuthiuron, além de testemunha, foram aplicados para o controle de Cynodon dactylon, Chloris polydactyla e Merremia aegyptia e sobre as MPBs das variedades RB 867515, RB 966928 e CTC 9001. Avaliações de controle foram realizadas aos 15, 30 e 45 dias após aplicação (DAA) e de fitotoxicidade na cultura aos 15, 30, 45 e 60 DAA, além da biomassa seca da parte aérea. Para o C. polydactyla todos os tratamentos foram eficazes, para M. aegyptia os melhores tratamentos foram saflufenacil, metribuzin e saflufenacil + tebuthiuron e saflufenacil + tebuthiuron. No controle de $C$. dactylon destacaram-se clomazone, metribuzin, saflufenacil + clomazone e tebuthiuron. Os tratamentos indaziflam e indaziflam + metribuzin prejudicaram o crescimento inicial das MPBs das três variedades de cana-de-açúcar estudadas, portanto, apresentam limitação no uso em MPBs.
\end{abstract}

\section{ABSTRACT}

Pre-sprouting seedlings (MPB) of sugarcane arrive in the field with the aerial part and roots developed, being able to present sensitivity to herbicides and to change weed control management. Therefore, the objectives of this work were: determine the chemical control of Cynodon dactylon, Chloris polydactyla and Merremia aegyptia; and to determine the effect of the herbicides on pre-budded seedlings of different varieties of sugarcane. The experimental design was completely randomized with eleven treatments and four replicates. The herbicides saflufenacil, clomazone, metribuzim, tebuthiuron, indaziflam, saflufenacil + clomazone, saflufenacil + metribuzim, saflufenacil + tebuthiuron, indaziflam + metribuzin, indaziflam + tebuthiuron, and control were applied for the control of Cynodon dactylon, Chloris polydactyla and Merremia aegyptia and on MPBs varieties - RB 867515, RB 966928 and CTC 9001. Control evaluations were performed at 15, 30 and 45 days after application (DAA) and phytotoxicity in the culture at 15, 30, 45 and $60 \mathrm{DAA}$, in addition to biomass of the aerial part. For C. polydactyla all treatments were effective, for $M$. aegyptia the best treatments were saflufenacil, metribuzin and saflufenacil + tebuthiuron e saflufenacil + tebuthiuron. In the control of $C$. dactylon we could highlight clomazone, metribuzin, saflufenacil + clomazone e tebuthiuron. The indaziflam and indaziflam + metribuzin treatments impaired the initial growth of the MPBs of the three studied sugarcane varieties; therefore, they present limitations in the use of MPBs. 


\section{Introdução}

A interferência exercida pelas plantas daninhas é um dos principais fatores limitantes da produtividade em canade-açúcar, pois essas espécies competem por água, luz, nutriente e espaço, causando perdas significativas tanto na produtividade da cultura quanto na redução da longevidade dos canaviais. Podem também dificultar operações de colheita sendo ainda hospedeiras de pragas e doenças (KUVA et al., 2003; NEGRISOLI et al., 2004; PROCÓPIO et al., 2004).

Dessa forma para minimizar as perdas, o controle químico acaba sendo o método mais utilizado, em razão das grandes áreas plantadas, da eficiência e do alto rendimento, aliado ao baixo custo em relação a outros métodos de controle e também por haver no mercado vários herbicidas registrados para essa cultura (PROCÓPIO et al., 2004; CHRISTOFFOLETI et al., 2004).

A planta daninha Chloris polydactyla (capimbranco), por exemplo, é bastante comum nas regiões Norte e Centro-Oeste, onde pode ser visto quase o ano todo em floração. Como planta daninha, é considerada medianamente frequente, encontrada principalmente em beira de estradas, pastagens e em pomares. Contudo, tem sido constatado o aumento da ocorrência dessa espécie nos últimos anos em lavouras perenes, inclusive em áreas cultivadas com cana-de-açúcar (LORENZI, 2014). Malardo et al. (2017) verificaram que o herbicida indaziflam aplicado isoladamente foi o único tratamento ineficaz para o controle desta planta daninha, por outro lado, indaziflam + metribuzin ou indaziflam + tebuthiuron foram eficazes no controle.

A planta daninha $C$. dacytlon (grama seda), por ser uma espécie de hábito rasteiro, interfere na cana-de-açúcar especialmente pela competição por água e nutrientes. Mesmo sem grande competição por luz, a produtividade da cana-de-açúcar pode ser reduzida em até $32 \%$ em convivência com a grama-seda (RICHARD; DALLEY, 2007). Esta planta daninha devido à presença de rizomas e estolões, além de apresentarem elevada capacidade reprodutiva é considerada de difícil controle tanto mecânico como químico (SANTOS et al., 2010; MACHADO et al., 2008).

Kuva et al. (2007) fizeram levantamento fitossociológico de comunidades infestantes em 28 áreas cultivas por cana-de-açúcar colhida mecanicamente (cana crua) e observaram as espécies do gênero Convolvulaceae em 17 áreas. Portanto, os herbicidas utilizados em áreas com cana crua precisam ter duas características fundamentais: transpor a palha sobre o solo e controlar as diferentes especies do gênero Ipomoea e Merremia. Toledo et al. (2017) verificaram que os herbicidas diuron + hexazione + sulfumeturon-methyl, amicarbazone, amicabazone + isoxaflutole, sufentrazone, imazapic; e tebuthiuron + isoxaflutole foram eficazes para as diferentes espécies de corda-de-viola (Ipomoea hederifolia, Ipomoea quamoclit e Merremia aegyptia) quando aplicados em cana-crua na época seca em solos areno-argilosos (textura média).
Entretanto, existem poucas informações a respeito sobre controle de plantas daninhas em área cultivadas com cana-de-açúcar pelo sistema de mudas pré-brotadas (MPBs). Este sistema é uma nova tecnologia para multiplicação em viveiros. São mudas provindas de gemas individualizadas oriundas de colmo-sementes, combinando elevado padrão de fitossanidade e vigor com ganhos econômicos na implantação de viveiros, replantio de áreas comerciais, introdução de materiais, possível renovação e expansão da cultura em outras áreas (LANDELL, 2013). Além de áreas de viveiros primários e secundários, a nova tecnologia vem sendo utilizada para o replantio de falhas de talhões plantados pelo método convencional e para replantio de áreas com cana soca.

O uso de herbicidas em MPB foi estudado por Sabbag et al. (2017) que verificaram a aplicação dos herbicidas ametryn, clomazone, hexazinone + diuron, metribuzin, sulfentrazone e tebuthiuron, isolados e em associação com saflufenacil aos 3 e 10 dias após o plantio (DAP) em mudas pré-brotadas de cana-de-açúcar. Os herbicidas foram seguros tanto aos 3 como aos 10 DAP na variedade RB867515, já as variedades RB 966928 e RB 975201 a aplicação aos 3 DAP causou menor fitotoxicidade, enquanto, para a RB 855156 foi aos 10 DAP.

Em outro trabalho, Rocha Neto (2015) observou que as mudas pré-brotadas da variedade IAC SP95-500 apresentaram leves sintomas de fitotoxicidade (10 a 20\%) e nenhum comprometimento na estatura de plantas com o uso dos herbicidas imazapyr aplicado aos 60 dias antes do plantio; imazapic aplicado aos 45 dias antes do plantio; trifluralin + pendimethalin, diclosulan + s-metolachlor e diclosulan + oxyfluorfen aplicados aos 30 dias antes do plantio; sulfentrazone; amicarbazone e tebuthiuron aplicados aos 2 dias antes do plantio.

Beluci et al. (2015) estudaram como os herbicidas pré-emergentes afetaram o desenvolvimento das MPBs da variedade IAC 95-5000. Constaram fitotoxicidade quando as mudas foram plantadas aos 20 e 40 dias após a aplicação do imazapyr e aos 20 dias após a aplicação de imazapic, diclosulam + s-metolachor e trifluralin + pendimenthalin.

Da mesma forma, Dias et al. (2017) observaram sintomas de fitotoxicidade em MPBs das variedades CTC 14, CTC 7 e RB 966928 com uso de sulfentrazone em préplantio, sendo que o mesmo resultado não foi encontrado com o uso dos herbicidas s-metolachlor, clomazone, diclosulan, metribuzin, diuron+hexazinone s s-metolachlor + sulfentrazone.

O uso de MPBs é um sistema de multiplicação que proporciona elevado padrão de fitossanidade, vigor e uniformidade de plantio, entretanto, há carência de informações sobre como os herbicidas podem interferir nos desenvolvimento das mudas. Portanto, os objetivos deste trabalho foram: determinar o controle químico das plantas daninhas Cynodon dactylon, Chloris polydactyla e Merremia aegyptia; e determinar o efeito dos herbicidas utilizados no controle das plantas daninhas em mudas de pré -brotadas de diferentes variedades de cana-de-açúcar: RB 86 7515, RB 966928 e CTC 9001. 


\section{Material e métodos}

O experimento foi realizado entre os meses de novembro de 2017 a maio de 2018 e conduzido em casa de vegetação, localizada nas coordenadas geográficas: latitude $22^{\circ} 18 ' 25,3^{\prime \prime}$ S, longitude $47^{\circ} 23^{\prime} 10,4^{\prime \prime}$ 'W e altitude média de $707 \mathrm{~m}$. A irrigação das plantas foi por aspersão controlada, $10 \mathrm{~mm}$ por dia de água. As unidades experimentais foram representadas por vasos com capacidade volumétrica de 10L, preenchidos com amostras de um Latossolo Vermelho Distrófico (Tabela 1). No experimento envolvendo as mudas pré-brotadas de cana-de-açúcar foi feita uma adubação com $30 \mathrm{~kg} \mathrm{ha}^{-1}$ de $\mathrm{N}, 80 \mathrm{~kg} \mathrm{ha}^{-1}$ de $\mathrm{P}_{2} \mathrm{O}_{5}$ e $80 \mathrm{~kg} \mathrm{ha}^{-1}$ de $\mathrm{K}_{2} \mathrm{O}$ e aos 45 dias após o plantio uma adubação de cobertura com $45 \mathrm{~kg} \mathrm{ha}^{-1}$ de N, de acordo com recomendação do Raij et al (1996).

Tabela 1. Características físico-químicas do solo utilizado nos experimentos.

\begin{tabular}{|c|c|c|c|c|c|c|c|c|}
\hline pH & $P$ res. & $\mathbf{K}$ & $\mathbf{C a}$ & Mg & M.O. & SB & СТC & $\mathbf{V}$ \\
\hline $\mathrm{CaCl}_{2}$ & $\mathrm{mg} / \mathrm{dm}^{-3}$ & \multicolumn{3}{|c|}{$----\mathbf{m m o l}_{\mathbf{c}} \mathbf{d m}^{-3}$} & $---\mathbf{g ~ d m}^{-3}--$ & \multicolumn{2}{|c|}{$\operatorname{mmol}_{c} \mathbf{d m}^{-3}$} & $\%$ \\
\hline 5,5 & 5,0 & 0,8 & 20,0 & 9,0 & 25,0 & 29,8 & 58,8 & 51,0 \\
\hline \multicolumn{2}{|c|}{ Argila } & \multicolumn{2}{|c|}{ Silte } & \multicolumn{2}{|r|}{ Areia Total } & \multicolumn{3}{|c|}{ Classe Textural } \\
\hline \multicolumn{2}{|c|}{520} & \multicolumn{2}{|c|}{330} & \multicolumn{2}{|r|}{150} & \multicolumn{3}{|c|}{ Média } \\
\hline
\end{tabular}

Foram realizados dois grupos de experimentos: um sobre controle de plantas daninhas e outro sobre efeito dos herbicidas sobre as mudas de cana-de-açúcar. Ambos os experimentos apresentaram delineamento inteiramente casualizado com onze tratamentos herbicidas e quatro repetições.

No experimento de controle, as semeaduras de Chloris polydactyla e Merremia aegyptia foram realizadas segundo a recomendação da empresa Agrocosmos, especialista em produção de sementes, com o intuito de obter quatro plantas por vaso. As quantidades de sementes utilizadas foram de $0,1 \mathrm{~g}$ para $C$. polydactyla e $0,5 \mathrm{~g}$ para $M$. aegyptia. No caso de $C$. dactylon, foram utilizados no plantio 8 estolões por vaso. Após um dia de semeadura/ plantio foram aplicados os tratamentos herbicidas.

No segundo experimento, os tratamentos com herbicidas foram aplicados aos sete dias após o transplante das MPBs das diferentes variedades de cana-de-açúcar RB 86 7515, RB 966928 e CTC 9001. No momento do plantio as mudas de cana-de-açúcar que vieram em tubetes foram caracterizadas com altura média de $14,0 \mathrm{~cm}, 0,50 \mathrm{~cm}$ de diâmetro e seis folhas e sem perfilho.

A aplicação dos tratamentos foi realizada via pulverizador costal propelido a $\mathrm{CO}_{2}$, equipado com barra com quatro pontas de pulverização, modelo XR 110.02, a uma pressão constante de 205,94 Kpa, propiciando um volume de calda de $200 \mathrm{~L} \mathrm{ha}^{-1}$, sendo que as pontas foram posicionadas a uma altura de $50 \mathrm{~cm}$ do alvo. No momento da aplicação a temperatura média era de $24^{\circ} \mathrm{C}$, umidade relativa de $60 \%$ e velocidade do vento de $3 \mathrm{~km} / \mathrm{h}$.

Os tratamentos foram: saflufenacil (Heat WG, Basf); clomazone (Gamit 360 CS, FMC), metribuzim (Sencor 480 SC, Bayer), tebuthiuron (Combine 500 SC, Corteva), indaziflam (Alion SC, Bayer). E as misturas saflufenacil + clomazone, saflufenacil + metribuzim, saflufenacil + tebuthiuron, indaziflam + metribuzin, indaziflam + tebuthiuron nas doses comerciais, além de testemunha sem aplicação dos herbicidas (Tabela 2).

Tabela 2. Tratamentos herbicidas utilizados nos experimentos, de controle e seletividade. Araras-SP.

\begin{tabular}{lcc}
\hline \multicolumn{1}{c}{ Tratamentos } & \multicolumn{2}{c}{ dose $-(\mathrm{L} \mathrm{ou} \mathrm{Kg} / \mathrm{ha})$} \\
\cline { 2 - 3 } & $\mathrm{pc}^{1}$ & i. $^{2}$ \\
\hline testemunha & & 0,075 \\
indaziflam & 0,15 & $0,075+1,680$ \\
indaziflam + metribuzin & $0,15+3,5$ & 1,680 \\
metribuzim & 3,5 & $0,098+0,7$ \\
saflufenacil+clomazone & $0,140+1,4$ & 0,8 \\
tebuthiuron & 1,6 & 0,098 \\
saflufenacil & 0,140 & $0,075+0,8$ \\
indaziflam + tebuthiuron & $0,15+1,6$ & $0,098+0,8$ \\
saflufenacil+tebuthiuron & $0,140+1,6$ & 0,7 \\
clomazone & 1,4 & $0,098+1,680$ \\
saflufenacil+metribuzim & $0,140+3,5$ & \\
\hline
\end{tabular}

${ }^{1} \mathrm{pc}=$ produto comercial; ${ }^{2}$ i.a. $=$ ingrediente ativo 
R. P. SILVA et al.

Para avaliar o controle de C. dactylon, C. polydactyla e M. aegyptia foram feitas avaliações visuais de controle aos 15,30 e 45 dias após a aplicação (DAA), seguindo a escala de notas de 0 a $100 \%$, onde 0 não houve controle e $100 \%$ houve morte total das plantas(ALAM, 1974). E na última avaliação as plantas daninhas foram coletadas para avaliação da biomassa seca da parte aérea, onde foram cortadas rente ao solo, acondicionadas e sacos de papel e identificas e levadas para secar em estufa com circulação de ar a $65^{\circ} \mathrm{C}$ até peso constante, e após foram pesadas.

As MPBs foram avaliadas quanto a fitotoxicidade aos $15,30,45$ e 60 DAA utilizando-se uma escala de notas de 0 a $100 \%$, onde $0 \%$ representa ausência de injurias e $100 \%$ à morte das plantas, respectivamente. Na última avaliação as plantas de cana-de-açúcar foram também avaliadas pela biomassa seca da parte aérea, conforme realizado com as plantas daninhas.

Os dados foram coletados e tabulados, e depois submetidos a análise de variância, e quando significativos, as médias foram comparadas pelo teste de Scott-Knott a 5\% de probabilidade usando o pacote Assistat 7.7.

\section{Resultados e discussão}

Com relação ao controle de $C$. dacytlon, é possível notar que os tratamentos, saflufenacil + clomazone, saflufenacil + tebuthiuron, saflufenacil + metribuzin e clomazone foram os mais eficientes com valores variando de 50 a $60 \%$, na avaliação de 15 DAA. Por outro lado, os tratamentos indaziflam e indaziflam + metribuzin, apresentaram os menores valores de controle com 5 e $13,75 \%$ respectivamente (Tabela 3 ).

Tabela 3. Controle (\%) de Cynodon dactylon aos 15, 30 e 45 DAA e biomassa seca da parte a aérea (g).

\begin{tabular}{|c|c|c|c|c|}
\hline \multirow[b]{2}{*}{ Tratamentos } & \multicolumn{3}{|c|}{ Controle $(\%)$} & \multirow{2}{*}{$\begin{array}{c}\text { Biomassa } \\
\text { (g) }\end{array}$} \\
\hline & $15 \mathrm{DAA}$ & $30 \mathrm{DAA}$ & $45 \mathrm{DAA}$ & \\
\hline $\begin{array}{l}\text { testemunha } \\
\text { indaziflam }\end{array}$ & $\begin{array}{l}0,00 \mathrm{c} \\
5,00 \mathrm{c}\end{array}$ & $\begin{array}{l}0,00 \mathrm{~d} \\
0,00 \mathrm{~d}\end{array}$ & $\begin{array}{l}0,00 \mathrm{~d} \\
0,00 \mathrm{~d}\end{array}$ & $\begin{array}{l}0,88 \mathrm{a} \\
1,08 \mathrm{a}\end{array}$ \\
\hline $\begin{array}{l}\text { indaziflam + metribuzin } \\
\text { metribuzin } \\
\text { saflufenacil + clomazone } \\
\text { tebuthiuron } \\
\text { saflufenacil } \\
\text { indaziflam + tebuthiuron } \\
\text { saflufenacil + tebuthiuron } \\
\text { clomazone }\end{array}$ & $\begin{array}{l}13,75 \mathrm{c} \\
42,50 \mathrm{~b} \\
50,00 \mathrm{a} \\
36,25 \mathrm{~b} \\
43,75 \mathrm{~b} \\
43,75 \mathrm{~b} \\
52,50 \mathrm{a} \\
60,00 \mathrm{a}\end{array}$ & $\begin{array}{c}0,00 \mathrm{~d} \\
78,75 \mathrm{~b} \\
88,75 \mathrm{a} \\
65,00 \mathrm{c} \\
55,00 \mathrm{c} \\
72,50 \mathrm{~b} \\
72,50 \mathrm{~b} \\
100,00 \mathrm{a}\end{array}$ & $\begin{array}{c}0,00 \mathrm{~d} \\
95,00 \mathrm{a} \\
95,00 \mathrm{a} \\
98,75 \mathrm{a} \\
50,00 \mathrm{c} \\
77,50 \mathrm{~b} \\
76,25 \mathrm{~b} \\
100,00 \mathrm{a}\end{array}$ & $\begin{array}{l}1,13 \mathrm{a} \\
0,04 \mathrm{~b} \\
0,13 \mathrm{~b} \\
0,05 \mathrm{~b} \\
0,85 \mathrm{a} \\
0,19 \mathrm{~b} \\
0,54 \mathrm{~b} \\
0,00 \mathrm{~b}\end{array}$ \\
\hline saflufenacil + metribuzin & $55,00 \mathrm{a}$ & 83,75 a & $91,25 \mathrm{a}$ & $0,17 \mathrm{~b}$ \\
\hline CV\% & 26,38 & 9,86 & 15,80 & 80,08 \\
\hline
\end{tabular}

Letras minúsculas iguais na coluna não diferem entre si pelo teste de Scott-Knott a 5\%.

Na avaliação de 30 DAA, destaca-se o tratamento com clomazone que provocou morte das plantas, ou seja, $100 \%$ de controle. Os tratamentos saflufenacil + clomazone e saflufenacil + metribuzin, embora estatisticamente iguais ao clomazone, não provocaram a morte das plantas, apresentando 88,75 e $83,75 \%$ de controle, respectivamente (Tabela 3).

Aos 45 DAA, podem-se destacar os tratamentos metribuzin, saflufenacil + clomazone, tebuthiuron, e saflufenacil + metribuzin, que proporcionaram controle entre 90 a 95\%, e clomazone com controle de $100 \%$. Em relação aos tratamentos menos eficientes no controle de $C$. dactylon, destaca-se o indaziflam e a mistura indaziflam + metribuzin $0 \%$ de controle (Tabela 3).

De acordo com as avaliações de controle aos 15, 30 e 45 DAA em $C$. dactlyon, analisando isoladamente cada tratamento, podem-se destacar os controles com o uso de metribuzin, saflufenacil + clomazone, tebuthiuron, saflufenacil + metribuzin e clomazone, os quais foram crescentes ao longo do tempo, possibilitando assim a eficácia no manejo das plantas daninhas. Os valores de controle são condizentes com os valores de biomassa, podendo destacar uma variação de $0,88 \mathrm{~g}$ para a testemunha e $0,04 \mathrm{~g}$ para o metribuzin ( $95 \%$ de controle de $C$. dactyon).

Outros herbicidas provocaram controle eficiente de C. dacytlon. Para Martini et al. (2002), os melhores resultados de controle desta espécie daninha foram obtidos com 2,5 kg e.a.ha ${ }^{-1}$ do glifosato potássico, que também garantiu menor brotação da planta daninha. No entanto, a dosagem de $2,10 \mathrm{~kg}$ e.a.ha ${ }^{-1}$ deste mesmo herbicida não foi menos eficaz que o sulfosato e o glifosato, com 1,98 e $2,16 \mathrm{~kg}$ e.a.ha ${ }^{-1}$, respectivamente.

No controle de C. polydactyla, apenas o tratamento indaziflam teve controle regular com $43,33 \%$ diferenciando dos demais tratamentos. Os tratamentos com saflufenacil + clomazone indaziflam + tebuthiuron e saflufenacil + metribuzin foram os únicos que provocaram $100 \%$ de controle logo na avaliação de 15 DAA. Já aos 30 DAA, foi possível notar que indaziflam + metribuzin, metribuzin, tebuthiuron, saflufenacil, saflufenacil + tebuthiuron, e clomazone também controlaram $100 \%$ da daninha (Tabela 4). 
R. P. SILVA et al.

Tabela 4. Controle (\%) de C. polydactyla aos 15, 30 e 45 DAA e biomassa seca da parte a aérea (g).

\begin{tabular}{|c|c|c|c|c|}
\hline \multirow[b]{2}{*}{ Tratamentos } & \multicolumn{3}{|c|}{ Controle (\%) } & \multirow{2}{*}{$\begin{array}{c}\text { Biomassa } \\
\text { (g) }\end{array}$} \\
\hline & $15 \mathrm{DAA}$ & $30 \mathrm{DAA}$ & 45 DAA & \\
\hline testemunha & $0,00 \mathrm{c}$ & $0,00 \mathrm{~b}$ & $0,00 \mathrm{~b}$ & $0,66 \mathrm{a}$ \\
\hline indaziflam & $43,33 \mathrm{~b}$ & $80,00 \mathrm{a}$ & $80,00 \mathrm{a}$ & $0,15 \mathrm{~b}$ \\
\hline indaziflam + metribuzin & $96,67 \mathrm{a}$ & $100,00 \mathrm{a}$ & $100,00 \mathrm{a}$ & $0,00 \mathrm{~b}$ \\
\hline metribuzin & $93,33 \mathrm{a}$ & $100,00 \mathrm{a}$ & $100,00 \mathrm{a}$ & $0,00 \mathrm{~b}$ \\
\hline saflufenacil +clomazone & $100,00 \mathrm{a}$ & $100,00 \mathrm{a}$ & $100,00 \mathrm{a}$ & $0,00 \mathrm{~b}$ \\
\hline tebuthiuron & $95,00 \mathrm{a}$ & $100,00 \mathrm{a}$ & $100,00 \mathrm{a}$ & $0,00 \mathrm{~b}$ \\
\hline saflufenacil & 98,33 a & $100,00 \mathrm{a}$ & $100,00 \mathrm{a}$ & $0,00 \mathrm{~b}$ \\
\hline indaziflam + tebuthiuron & $100,00 \mathrm{a}$ & $100,00 \mathrm{a}$ & $100,00 \mathrm{a}$ & $0,00 \mathrm{~b}$ \\
\hline saflufenacil + tebuthiuron & $95,00 \mathrm{a}$ & $100,00 \mathrm{a}$ & $100,00 \mathrm{a}$ & $0,00 \mathrm{~b}$ \\
\hline clomazone & $96,67 \mathrm{a}$ & $100,00 \mathrm{a}$ & $100,00 \mathrm{a}$ & $0,00 \mathrm{~b}$ \\
\hline saflufenacil + metribuzin & $100,00 \mathrm{a}$ & $100,00 \mathrm{a}$ & $100,00 \mathrm{a}$ & $0,00 \mathrm{~b}$ \\
\hline $\mathrm{CV} \%$ & 20,18 & 11,84 & 18,68 & 47,20 \\
\hline
\end{tabular}

Letras minúsculas iguais na coluna não diferem entre si pelo teste de Scott-Knott a 5\%.

$\mathrm{Na}$ terceira avaliação, o tratamento indaziflam permaneceu com $80 \%$ de controle, sendo o único tratamento que não controlou totalmente a $C$. polydactyla, entretanto, ainda dentro dos valores mínimos de controle $(80 \%)$. Valores de biomassa seca da parte aérea que foram encontrados são condizentes com os valores de controle (Tabela 4). Malardo et al. (2017) verificaram a eficiência dos herbicidas metribuzin, tebuthiuron, indaziflam + metribuzin e indaziflam + tebuthiuron, aplicados em préemergência sobre quatro quantidades de palha $(0 ; 1 ; 2$ e $4 \mathrm{t}$ $\mathrm{ha}^{-1}$ ) no controle desta planta daninha.
De acordo com a tabela 5 , foi possível notar que os tratamentos indaziflam, metribuzin, saflufenacil + clomazone, saflufenacil, saflufenacil + tebuthiuron e saflufenacil + metribuzin foram os tratamentos com melhores resultados e estaticamente iguais na avaliação controle aos 15 DAA da espécie $M$. aegyptia. Em contrapartida, os tratamentos indaziflam + metribuzin, tebuthiuron, indaziflam + tebuthiuron e clomazone foram os que proporcionaram controle com valores que variaram de 21,67 a $40 \%$.

Tabela 5. Controle (\%) de Merremia aegyptia aos 15, 30 e 45 DAA e biomassa seca da parte a aérea (g).

\begin{tabular}{|c|c|c|c|c|}
\hline \multirow[b]{2}{*}{ Tratamentos } & \multicolumn{3}{|c|}{ Controle $(\%)$} & \multirow{2}{*}{$\begin{array}{c}\text { Biomassa } \\
(\mathrm{g})\end{array}$} \\
\hline & $15 \mathrm{DAA}$ & $30 \mathrm{DAA}$ & $45 \mathrm{DAA}$ & \\
\hline $\begin{array}{l}\text { testemunha } \\
\text { indaziflam }\end{array}$ & $\begin{array}{c}0,00 \mathrm{c} \\
73,33 \mathrm{a}\end{array}$ & $\begin{array}{c}0,00 \mathrm{~d} \\
76,67 \mathrm{~b}\end{array}$ & $\begin{array}{c}0,00 \mathrm{c} \\
73,33 \mathrm{~b}\end{array}$ & $\begin{array}{l}0,43 \mathrm{a} \\
0,16 \mathrm{a}\end{array}$ \\
\hline $\begin{array}{l}\text { indaziflam + metribuzin } \\
\text { metribuzin } \\
\text { saflufenacil +clomazone } \\
\text { tebuthiuron } \\
\text { saflufenacil } \\
\text { indaziflam + tebuthiuron } \\
\text { saflufenacil + tebuthiuron } \\
\text { clomazone }\end{array}$ & $\begin{array}{l}21,67 \mathrm{~b} \\
93,33 \mathrm{a} \\
93,33 \mathrm{a} \\
23,33 \mathrm{~b} \\
96,67 \mathrm{a} \\
40,00 \mathrm{~b} \\
96,67 \mathrm{a} \\
33,33 \mathrm{~b}\end{array}$ & $\begin{array}{c}25,00 \mathrm{c} \\
93,33 \mathrm{a} \\
93,33 \mathrm{a} \\
33,33 \mathrm{c} \\
100,00 \mathrm{a} \\
63,33 \mathrm{~b} \\
100,00 \mathrm{a} \\
33,33 \mathrm{c}\end{array}$ & $\begin{array}{c}10,00 \mathrm{c} \\
100,00 \mathrm{a} \\
80,00 \mathrm{~b} \\
10,00 \mathrm{c} \\
100,00 \mathrm{a} \\
60,00 \mathrm{~b} \\
100,00 \mathrm{a} \\
20,00 \mathrm{c}\end{array}$ & $\begin{array}{l}0,36 \mathrm{a} \\
0,01 \mathrm{~b} \\
0,11 \mathrm{a} \\
0,42 \mathrm{a} \\
0,00 \mathrm{~b} \\
0,26 \mathrm{a} \\
0,01 \mathrm{~b} \\
0,44 \mathrm{a}\end{array}$ \\
\hline saflufenacil + metribuzin & 93,33 a & $96,67 \mathrm{a}$ & $98,33 \mathrm{a}$ & $0,03 \mathrm{~b}$ \\
\hline $\mathrm{CV} \%$ & 20,16 & 18,61 & 32,28 & 88,17 \\
\hline
\end{tabular}

Letras minúsculas iguais na coluna não diferem entre si pelo teste de Scott-Knott a 5\%.

Aos 30 DAA os tratamentos mais eficazes foram metribuzin, saflufenacil + clomazone, saflufenacil, saflufenacil + tebuthiuron e saflufenacil + metribuzin, com valores de 93,33\%, 93,33\%, 100\%, 100\%, 96,67\%, respectivamente (Tabela 5).

Aos 45 DAA, o tratamento saflufenacil + metribuzin resultou em faixas próximas aos $100 \%$ de controle da daninha e sem diferença estatística com os tratamentos saflufenacil, metribuzin e saflufenacil + tebuthiuron que obtiveram controle total da daninha, que também refletiu nos valores de biomassa da parte aérea. Para os tratamentos indaziflam + metribuzin, tebuthiuron e clomazone, os valores de controle de $M$. aegyptia, decresceram se comparados com as primeiras avaliações, resultando assim em tratamentos com pouca eficácia para o controle da $M$. aegyptia.

Em área de cana-de-açúcar, Silva et al. (2015) trabalhando com herbicidas sulfentrazone (800 $\left.\mathrm{g} \mathrm{ha}^{-1}\right)$, 
R. P. SILVA et al.

hexazinone $\left(187,5 \mathrm{~g} \mathrm{ha}^{-1}\right)$, amicarbazone $\left(1050 \mathrm{~g} \mathrm{ha}^{-1}\right)$ e suas associações em diferentes doses, verificaram que a espécie M. aegyptia foi controlada eficientemente com os herbicidas amicarbazone $(99,99 \%)$ e sulfentrazone $(88,00 \%)$ e, com menor eficiência pelo hexazinone $(75 \%)$. Correia et al. (2010) também observaram eficiência no controle desta planta daninha com uso de amicarbazone.

Aos 15 DAA na RB 867515, todos os tratamentos causaram sintomas de fitotoxicidade na cultura, variando de $26,67 \%$ com uso de metribuzin a $66,67 \%$ nos tratamentos indaziflam e indaziflam + metribuzin, o que poderia comprometer o crescimento inicial da planta e o fechamento da entrelinha. Aos 30 DAA os tratamentos envolvendo clomazone e saflufenacil + clomazone apresentaram redução na fitotoxicidade (Tabela 6).

Tabela 6. Fitotoxicidade aos 15, 30, 45 e 60 DAA dos tratamentos e biomassa seca da parte aérea (g) das plantas de cana de açúcar, variedade RB 867515.

\begin{tabular}{|c|c|c|c|c|c|}
\hline \multirow[b]{2}{*}{ Tratamentos } & \multicolumn{3}{|c|}{ Fitotoxicidade (\%) } & \multirow[b]{2}{*}{60 DAA } & \multirow{2}{*}{$\begin{array}{c}\text { Biomassa } \\
\text { (g) }\end{array}$} \\
\hline & 15 DAA & $30 \mathrm{DAA}$ & $45 \mathrm{DAA}$ & & \\
\hline testemunha & $0,00 \mathrm{c}$ & $0,00 \mathrm{c}$ & $0,00 \mathrm{~d}$ & $0,00 \mathrm{c}$ & $20,00 \mathrm{a}$ \\
\hline indaziflam & $66,67 \mathrm{a}$ & 73,33 a & $90,00 \mathrm{a}$ & $100,00 \mathrm{a}$ & $0,00 \mathrm{~b}$ \\
\hline indaziflam + metribuzin & $66,67 \mathrm{a}$ & $70,00 \mathrm{a}$ & $66,67 \mathrm{~b}$ & 85,00 a & $11,55 \mathrm{~b}$ \\
\hline metribuzin & $26,67 \mathrm{~b}$ & $0,00 \mathrm{c}$ & $0,00 \mathrm{~b}$ & $0,00 \mathrm{c}$ & $19,47 a b$ \\
\hline saflufenacil +clomazone & $33,33 \mathrm{~b}$ & $10,00 \mathrm{c}$ & $0,00 \mathrm{~d}$ & $0,00 \mathrm{c}$ & $27,22 \mathrm{a}$ \\
\hline tebuthiuron & $30,00 \mathrm{~b}$ & $0,00 \mathrm{c}$ & $0,00 \mathrm{~d}$ & $0,00 \mathrm{c}$ & $23,70 \mathrm{a}$ \\
\hline saflufenacil & $33,33 \mathrm{~b}$ & $0,00 \mathrm{c}$ & $0,00 \mathrm{~d}$ & $0,00 \mathrm{c}$ & $24,33 \mathrm{a}$ \\
\hline indaziflam + tebuthiuron & $43,33 \mathrm{~b}$ & $53,33 \mathrm{~b}$ & $30,00 \mathrm{c}$ & $53,33 \mathrm{~b}$ & $14,54 \mathrm{~b}$ \\
\hline saflufenacil + tebuthiuron & $36,67 \mathrm{~b}$ & $0,00 \mathrm{c}$ & $0,00 \mathrm{~d}$ & $0,00 \mathrm{c}$ & $16,32 a b$ \\
\hline clomazone & $30,00 \mathrm{~b}$ & $3,33 \mathrm{c}$ & $0,00 \mathrm{~d}$ & $0,00 \mathrm{c}$ & $26,22 \mathrm{a}$ \\
\hline saflufenacil + metribuzin & $43,33 \mathrm{~b}$ & $3,33 \mathrm{c}$ & $0,00 \mathrm{~d}$ & $0,00 \mathrm{c}$ & $21,98 \mathrm{a}$ \\
\hline $\mathrm{CV} \%$ & 22,40 & 35,90 & 51,29 & 38,32 & 22,40 \\
\hline
\end{tabular}

Letras minúsculas iguais na coluna não diferem entre si pelo teste de Scott Knott a 5\%.

Costa (2012) observaram que o saflufenacil, associado ao clomazone, provocou fitotoxicidade de cerca de 30\%, quando aplicado na RB 867515 e RB 966928 e esse potencial fitotóxico foi confirmado por Inoue et al. (2007) que trabalhando com a variedade RB 867515, acabou encontrando valores parecidos.

Aos 30 e 45 DAA observa-se novamente o aumento na fitotoxicidade nos tratamentos indaziflam $(73,33$ e $90 \%$, respectivamente), indaziflam + metribuzin (70 e 66,67\%) e indaziflam + tebuthiuron $(53,33$ e $30 \%)$. Já na última avaliação, os tratamentos indaziflam e indaziflam + metribuzin se mostraram estatisticamente iguais e diferentes dos demais e os mais prejudiciais ao desenvolvimento da RB 867515, com fitotoxicidade de $100 \%$ e $85 \%$, respectivamente (Tabela 6 ).

Os mesmos resultados de 60 DAA foram obtidos no trabalho de Hijano (2016), para as cultivares RB 966928 e CTC 14, em pós-plantio utilizando a dose de $37,5 \mathrm{~g}^{\text {i.a }} \mathrm{ha}^{-1}$ de indaziflam. Para indaziflam + metribuzin a aplicação em pré-plantio também ocasionou a morte das mudas das duas cultivares testadas, mas foi seletivo para aplicação em pósplantio nas doses de 37,5 + 480720 ; e 75+960 g i.a ha ${ }^{-1}$.

Para os resultados de fitotoxicidade entre as datas de aplicação, alguns tratamentos como metribuzin, tebuthiuron, saflufenacil e saflufenacil + tebuthiuron provocaram $0 \%$ de fitotoxicidade na segunda avaliação, mostrando assim recuperação rápida.

Com relação a produção de biomassa seca da parte aérea, os tratamentos indaziflam, indaziflam + metribuzin e indaziflam + tebuthiuron proporcionaram os menores valores de biomassa seca da parte aérea das plantas de canade-açúcar com 0,0; 11,55 e 14,54g, respectivamente (Tabela $6)$.

Os resultados de fitotoxicidade sobre as plantas de cana de açúcar da variedade RB 966928 estão apresentados na tabela 7. Constatou-se que, aos 15 DAA, os valores de fitotoxicidade foram de $20 \%$ para indaziflam + tebuthiuron a $43,33 \%$ para indaziflam + metribuzin. 
R. P. SILVA et al.

Tabela 7. Fitotoxicidade em 15, 30, 45 e 60 DAA dos tratamentos e biomassa seca da parte a aérea (g) das plantas de cana de açúcar, variedade RB 966928.

\begin{tabular}{|c|c|c|c|c|c|}
\hline \multicolumn{5}{|c|}{ Fitotoxicidade (\%) } & \multirow{2}{*}{$\begin{array}{c}\text { Biomassa } \\
\text { (g) }\end{array}$} \\
\hline Tratamentos & $15 \mathrm{DAA}$ & $30 \mathrm{DAA}$ & $45 \mathrm{DAA}$ & $60 \mathrm{DAA}$ & \\
\hline testemunha & $0,00 \mathrm{c}$ & $0,00 \mathrm{a}$ & $0,00 \mathrm{~b}$ & $0,00 \mathrm{~d}$ & $28,12 \mathrm{a}$ \\
\hline indaziflam & $33,33 \mathrm{~b}$ & $0,00 \mathrm{a}$ & 46,66 a & $80,00 \mathrm{a}$ & 20,03 a \\
\hline indaziflam + metribuzin & $43,33 \mathrm{a}$ & $0,00 \mathrm{a}$ & $0,00 \mathrm{~b}$ & $60,00 \mathrm{a}$ & $22,06 \mathrm{a}$ \\
\hline metribuzin & $40,00 \mathrm{ab}$ & $0,00 \mathrm{a}$ & $0,00 \mathrm{~b}$ & $0,00 \mathrm{~d}$ & $31,17 \mathrm{a}$ \\
\hline saflufenacil+clomazone & $30,00 \mathrm{~b}$ & $0,00 \mathrm{a}$ & $0,00 \mathrm{~b}$ & $0,00 \mathrm{~d}$ & $27,29 \mathrm{a}$ \\
\hline tebuthiuron & $30,00 \mathrm{~b}$ & $0,00 \mathrm{a}$ & $0,00 \mathrm{~b}$ & $0,00 \mathrm{~d}$ & $32,77 \mathrm{a}$ \\
\hline saflufenacil & $23,33 \mathrm{bc}$ & $3,33 \mathrm{a}$ & $0,00 \mathrm{~b}$ & $0,00 \mathrm{~d}$ & $33,94 \mathrm{a}$ \\
\hline indaziflam +tebuthiuron & $20,00 \mathrm{bc}$ & $0,00 \mathrm{a}$ & $0,00 \mathrm{~b}$ & $16,67 \mathrm{c}$ & $32,10 \mathrm{a}$ \\
\hline saflufenacil+tebuthiuron & $23,33 \mathrm{bc}$ & $0,00 \mathrm{a}$ & $0,00 \mathrm{~b}$ & $33,33 \mathrm{~b}$ & $33,64 \mathrm{a}$ \\
\hline clomazone & $30,00 \mathrm{~b}$ & $3,33 \mathrm{a}$ & $0,00 \mathrm{~b}$ & $6,66 \mathrm{~d}$ & $36,65 \mathrm{a}$ \\
\hline saflufenacil + metribuzin & $30,00 \mathrm{~b}$ & $0,00 \mathrm{a}$ & $0,00 \mathrm{~b}$ & 56,66 a & $25,23 \mathrm{a}$ \\
\hline CV\% & 20,71 & 48,20 & 77,12 & 67,18 & 23,86 \\
\hline
\end{tabular}

Letras minúsculas iguais na coluna não diferem entre si pelo teste de Scott-Knott a 5\%.

$\mathrm{Na}$ avaliação realizada aos $30 \mathrm{DAA}$ e $45 \mathrm{DAA}$, as plantas se recuperaram da fitotoxicidade, apenas $\mathrm{o}$ indaziflam provocou fitotoxicidade de $46 \%$ aos 45 DAA. Na última avaliação, os tratamentos indaziflam, indaziflam + metribuzin e saflufenacil + metribuzin se mostraram estatisticamente iguais $\mathrm{e}$ os mais prejudiciais ao desenvolvimento da RB 966928, com fitotoxicidade de $80 \%, 60 \%$ e $56,66 \%$, respectivamente (Tabela 7 ). No caso, dos tratamentos com envolvendo o indaziflam, o aumento da fitotoxicidade ao longo do tempo, pode estar ligado a movimentação do herbicida indaziflam que possui alto residual para as camadas mais profundas no vaso e o posterior crescimento das raízes entrando em contato com o produto químico.

Para o saflufenacil, foi observado que misturas com clomazone, apresentam fitotoxicidade acima de $30 \%$ aos 15 DAA nas variedades SP80-1816 e RB 855156 (COSTA, 2012). Um resultado parecido foi observado no experimento logo na primeira avaliação 15 DAA, na variedade RB 966928 com $30 \%$ de controle e posterior recuperação das plantas.

Observa-se que os tratamentos não provocaram impacto na biomassa seca da parte aérea. Semelhante a este resultado, Sabbag et al. (2017) verificaram que na variedade RB 966928 ocorrem diferenças no parâmetro diâmetro e altura das plantas de cana-de-açúcar, porém não se notou diferenças nos valores de biomassa seca da parte aérea, com uso de indaziflam e indaziflam + metribuzin, tratamentos com maiores valores de fitotoxicidade sobre as mudas.

Com relação à resposta da CTC 9001 aos herbicidas, verificou-se que todos os tratamentos causaram sintomas aos 15 DAA, com valores que variaram de $20 \%$ para saflufenacil; indaziflam + tebuthiuron até $36,66 \%$ para indaziflam, com destaque para indaziflam, metribuzin, saflufenacil + clomazone, clomazone e saflufenacil + metribuzin, apresentando diferenças estatísticas (Tabela 8).

Tabela 8. Fitotoxicidade em 15, 30, 45 e 60 DAA dos tratamentos e biomassa seca da parte a aérea (g) das plantas de cana de açúcar, variedade CTC 9001.

\begin{tabular}{lcccc|c}
\hline & \multicolumn{2}{c|}{ Fitotoxicidade (\%) } & & $\begin{array}{c}\text { Biomassa } \\
(\mathrm{g})\end{array}$ \\
\cline { 1 - 4 } \multicolumn{1}{c}{ Tratamentos } & $15 \mathrm{DAA}$ & $30 \mathrm{DAA}$ & $45 \mathrm{DAA}$ & $60 \mathrm{DAA}$ & \\
\hline testemunha & $0,00 \mathrm{c}$ & $0,00 \mathrm{~b}$ & $0,00 \mathrm{c}$ & $0,00 \mathrm{c}$ & $17,45 \mathrm{a}$ \\
indaziflam & $33,300 \mathrm{a}$ & $20,00 \mathrm{a}$ & $40,00 \mathrm{a}$ & $81,66 \mathrm{a}$ & $12,03 \mathrm{~b}$ \\
indaziflam + metribuzin & $23,33 \mathrm{~b}$ & $0,00 \mathrm{~b}$ & $20,00 \mathrm{~b}$ & $40,00 \mathrm{~b}$ & $12,95 \mathrm{~b}$ \\
metribuzin & $33,33 \mathrm{a}$ & $0,00 \mathrm{~b}$ & $0,00 \mathrm{c}$ & $0,00 \mathrm{c}$ & $14,64 \mathrm{ab}$ \\
saflufenacil +clomazone & $33,33 \mathrm{a}$ & $10,00 \mathrm{a}$ & $0,00 \mathrm{c}$ & $0,00 \mathrm{c}$ & $14,51 \mathrm{ab}$ \\
tebuthiuron & $23,33 \mathrm{~b}$ & $0,00 \mathrm{~b}$ & $0,00 \mathrm{c}$ & $0,00 \mathrm{c}$ & $13,74 \mathrm{ab}$ \\
saflufenacil & $20,00 \mathrm{~b}$ & $0,00 \mathrm{~b}$ & $0,00 \mathrm{c}$ & $0,00 \mathrm{c}$ & $17,77 \mathrm{a}$ \\
indaziflam + tebuthiuron & $20,00 \mathrm{~b}$ & $0,00 \mathrm{~b}$ & $0,00 \mathrm{c}$ & $0,00 \mathrm{c}$ & $15,12 \mathrm{a}$ \\
saflufenacil + tebuthiuron & $23,33 \mathrm{~b}$ & $0,00 \mathrm{~b}$ & $0,00 \mathrm{c}$ & $0,00 \mathrm{c}$ & $14,06 \mathrm{ab}$ \\
clomazone & $30,00 \mathrm{a}$ & $0,00 \mathrm{~b}$ & $0,00 \mathrm{c}$ & $0,00 \mathrm{c}$ & $14,61 \mathrm{ab}$ \\
saflufenacil + metribuzin & $30,00 \mathrm{a}$ & $0,00 \mathrm{~b}$ & $0,00 \mathrm{c}$ & $0,00 \mathrm{c}$ & $15,36 \mathrm{a}$ \\
\multicolumn{1}{c}{$\mathrm{CV} \%$} & & & & \\
\hline
\end{tabular}

Letras minúsculas iguais na coluna não diferem entre si pelo teste de Scott-Knott a 5\%. 
$\mathrm{Na}$ avaliação realizada aos $30 \mathrm{DAA}$, todos os tratamentos apresentaram queda em fitotoxicidade. Aos 45 DAA, o tratamento que provocou maior fitoxicidade nas plantas foi o indaziflam com $40 \%$ de fitotoxicidade, com posterior evolução, sendo que aos 60 DAA a fitotoxicidade observada foi de $81,66 \%$ (Tabela 8 ).

Para os valores de biomassa seca da parte aérea das plantas de cana-de-açúcar, CTC 9001, é possível verificar menor acumulo nas plantas submetidas ao tratamento indaziflam+metribuzin com $12,95 \mathrm{~g}$ e maior acumulo no tratamento saflufenacil com 17,77g (Tabela 8).

O herbicida saflufenacil, utilizado de maneira isolada, e independente da variedade, não apresentou fitotoxicidade maior que o limite aceitável, ou seja, suas porcentagens foram abaixo de $30 \%$ em todas as variedades utilizadas, RB 867515, RB 966928 e CTC 9001. O que também é observado por (COSTA, 2012) onde o saflufenacil aplicado isoladamente apresentou fitotoxicidade menor que 20\%, nas variedades SP83-2847, SP80-3280, RB 85 5453, SP80-1842, SP89-1115, RB 86 7515, RB 855156 e SP81-3250.

\section{Conclusões}

Os tratamentos clomazone, metribuzin, saflufenacil + clomazone e tebuthiuron controlaram C. dactylon. Todos os tratamentos foram eficientes no controle de $C$. polydactyla. Para M aegyptia destacaram-se positivamente o uso de saflufenacil, metribuzin e saflufenacil + tebuthiuron e saflufenacil + tebuthiuron.

Os tratamentos indaziflam e indaziflam + metribuzin prejudicaram o crescimento inicial das MPBs das variedades de cana-de-açúcar RB 867515, RB 966928 e CTC 9001 e o tratamento saflufenacil + metribuzin para a RB 966928.

\section{Referências}

Association Latino americana De Malezas. Recomendaciones sobre unificación de los sistemas de evaluación em ensayos de control de malezas. Bogotá, ALAM, 1974 v. $\quad 1, \quad$ p. 35-38.

Beluci, L. R.; Vitorino, R.; Azania, C. A. M.; Azania, A. A. P. M.; Tortorelli, H. F.; Utilização do perfil isoenzimático como ferramenta na avaliação da seletividade de herbicidas em cana-de-açúcar. Nucleus, v.12, n.2, p. 157-166. 2015.

Correia, N. M.; Braz, B. A.; Fuzita, W. E. Eficácia de herbicidas aplicados nas épocas seca e úmida para o controle de Merremia aegyptia na cultura da cana-de-açúcar. Planta Daninha, v. 28, n. 3, p. 631-642, 2010.

Christoffoleti, P. J.; Lopez Ovejero, R. F.; Nicolai, M. Manejo de plantas daninhas. Atualidades Agrícolas, p. 1014,2004

Costa, S. I. A. Seletividade do herbicida saflufenacil aplicado em pós-emergência em dez variedades de cana- de-açúcar na condição de soca. São Paulo, Arq. Inst. Biol., 2012 v. 79, n. 1, p.113-120. 2012.

Dias, J.L.C.S.; Silva Junior, A.C.; Queiroz, J.R.G.; Martins, D. Herbicides selectivity in pre-budded seedlings of sugarcane. Arquivos do Instituto Biológico, v.84, n.e0112015, 2017.

Hijano, N. Interferência de capim-camalote em cana-deaçúcar e seletividade de indaziflam e indaziflam+ metribuzin aplicados em cana-de-açúcar no sistema MPB. 201611212 f. Tese (Mestrado em Agronomia) - Faculdade de Ciências Agrárias e Veterinárias - Unesp, Campus de Jaboticabal. 2016.

Inoue, M. H.; Santin, A. J.; Dallacort, R.; Possamai, A.S.; Santana, D.C, Performance de associações de herbicidas em cana-de-açúcar (Saccharum officinarum). Revista Brasileira de Herbicidas, v.6, n.2, p.32-41. 2007.

Kuva, M. A.; Gravena, R.; Pitelli, R. A.; Christoffoleti, P. J.; Alves, P. L. C. A. Períodos de interferência das plantas daninhas na cultura da cana de açúcar. III - capim braquiária (Brachiaria decumbens) e capim-colonião (Panicum maximum). Revista Planta Daninha, v. 21, n. 1, p. 37-44. 2003.

Kuva, M.; Pitelli, R. A.; Salgado, T. P.; Alves, P. L. C. A. Fitossociologia de comunidades de plantas daninhas em agroecossistema cana-crua. Planta Daninha, v. 25, n. 3, p. 501-511, 2007

Landell, M. G. A.; Campana, M. P.; Figueiredo, P.; Xavier, M. A. Sistema de Multiplicação de cana-de-açúcar com uso de mudas pré-brotadas (MPB), oriundas de gemas individualizadas. IAC, Campinas, 2013 109. ed. 16 p.

Lorenzi, H, manual de identificação e controle de plantas daninhas: plantio direto e convencional. Nova Odessa, Instituto Plantarum, 2014, 7.ed. 383 p.

Malardo, M.R.; Monquero, P.A.; Dos Santos, P.H.V.; Ribeiro, N.M.; Silva, P.V.; Hirata, A.C.S. Influence of the sowing depth and amount of sugarcane straw on the emergence of Chloris polydactyla and Eleusine indica and their control by herbicides applied pre-emergence. Semina: Ciências Agrárias, v. 38, n. 3, p. 1187-1200, maio/jun. 2017.

Machado, A.F.L.; Meira, R.M.S.; Ferreira, L.R.; Ferreira, F.A.; Tuffi Santos, L.D.; Teixeira Filho, C.M.; Machado, M.S. Caracterização anatômica de folha, colmo e rizoma de Digitaria insularis. Planta Daninha, v.26, n.1, p.1-8, 2008.

Martini, G.. Pedrinho Junior, A.F.F.; Felici, G.V.; Piva, F.M.; Durigan, J.C. Eficácia de uma nova formulação de glifosato para o controle de grama-seda (Cynodon dactylon), em pomar de citros. Rev. Bras. Frutic., v. 24, n. 3, p. 683686,2002 
Negrisoli, E.; Velini, E. D.; Tofoli, G. R.; Cavenaghi, A. L.; Martins, D.; Morelli, J. L.; Costa, A. G. F. Seletividade de herbicidas aplicados em pré-emergência na cultura da canade-açúcar tratada com nematicidas. Revista Planta Daninha, v. 22, n. 4, p. 567-575. 2004.

Procópio, S. O.; Silva, A. A.; Vargas, L.; Ferreira, F. A, Manejo de plantas daninhas na cultura da cana-deaçúcar. Viçosa: Suprema, 2004150 p.

Raij, B. Van; Cantarella, H.; Quaggio, J.A.; Furlani, A.M.C. (Ed.). Recomendações de adubação e calagem para o Estado de São Paulo. 2.ed. Campinas : Instituto Agronômico, 1996. 285p. (IAC. Boletim técnico, 100).

Richard Jr, E.P.; Dalley, C.D. Sugarcane response to bermudagrass interference. Weed Technology, v.21, p.941946, 2007.

Rocha Neto, A. R. da; Azania, C. A. M.; Borges, I. S.; Vitorino, R.; Marchine, N. M.; Azania, A. P. M. Tolerância de mudas pré brotadas de cana-de-açúcar a herbicidas aplicados em pré plantio. IX Workshop Agroenergia. 2015 Ribeirão Preto. Disponível em: <http://www.infobibos.com/ agroenergia/cd/Resumos/ResumoAgroenergia_2015_082 .pdf> Acesso em: set. 2018.

Sabbag. R.; Monquero, P. A.; Hirata A. C. S.; Santos, P. H. V. Crescimento inicial de mudas pré brotadas de cana-deaçúcar submetidas a aplicação de herbicidas. Revista Brasileira de Herbicidas, v.16, n.1, p.38-49. 2017

Santos, M.V.; Freitas, F.C.L.; Ferreira, F.A.; Carvalho, A.J.; Braz, T.G.S.; Cavali, J.; Rodrigues, O.L. Tolerância do Tifton 85 ao glyphosate em diferentes épocas de aplicação. Planta Daninha, v.28, n.1, p.131-137, 2010.

Silva, P.V., Monquero, P.A; Munhoz W.S. Controle em pósemergência de plantas daninhas por herbicidas utilizados na cultura da cana-de-açúcar. Revista Caatinga, v. 28, n. 4, p.21-32, out.-dez., 2015.

Toledo, R. E. B.; Victória Filho, R.; Negrisoli, E.; Correa, M.R. Manejo de cordas-de-violas (Ipomea hederifolia, Ipomea nil e Merremia aegyptia) com herbicidas em cana crua na época seca. Revista Brasileira de Herbicida, v. 16, n.2, p.84-90, 2017. 